\title{
Geolocalização de casos de sobrepeso/obesidade e pressão arterial alterada em escolares
}

\author{
Geolocation of overweight/obesity and abnormal \\ blood pressure cases among schoolchildren
}

Luiza Pasa, ${ }^{1}$ Lia Gonçalves Possuelo, ${ }^{1}$ Tássia Silvana Borges, ${ }^{2}$ Letícia de Borba Schneiders, ${ }^{1}$ Cristiane Fernanda da Silva, ${ }^{1}$ Letícia Welser, ${ }^{1}$ Deise Graziela Kern, ${ }^{1}$ Cézane Priscila Reuter, ${ }^{1}$ Miria Suzana Burgos

'Universidade de Santa Cruz do Sul (UNISC), Santa Cruz do Sul, RS, Brasil.

¿Universidade Luterana do Brasil (ULBRA), Canoas, RS, Brasil.

Recebido em: 23/05/2017 / Aceito em: 14/06/2017 / Publicado em: 30/06/2017

mburgos@unisc.br

\section{RESUMO}

Objetivo: descrever a geolocalização de sobrepeso/obesidade e pressão arterial alterada de escolares residentes em Santa Cruz do Sul/RS. Método: estudo transversal, incluindo 1578 escolares de ambos os sexos, com idade entre sete e 17 anos, de 18 escolas do município. Utilizou-se o índice de massa corporal para avaliar o sobrepeso/obesidade e, para pressão arterial, utilizou-se medida da pressão sistólica (PAS) e da pressão diastólica (PAD). Os mapas de geolocalização foram separados por sexo e escola e plotados no mapa de acordo com as variáveis avaliadas. Utilizou-se o programa SPSS 20.0, empregando estatística descritiva, teste qui-quadrado e teste $t$, considerando diferenças significativas para $p<0,05$. Para a confecção dos mapas, foi utilizado o programa Terraview 3.5.0 e CoreIDRAW X5. Resultados: observou-se prevalência de sobrepeso/obesidade de $27,2 \%$ nos meninos e $26,5 \%$ nas meninas. PAS alterada ocorreu em $14,8 \%$ dos meninos e $13,6 \%$ das meninas e PAD alterada em $12,7 \%$ dos meninos e $12,5 \%$ das meninas. A geolocalização apontou duas escolas da zona rural das regiões leste e oeste e uma escola da zona urbana na região central do município, com a maior prevalência de sobrepeso/obesidade, sendo que na escola rural da região leste mais de $50 \%$ dos escolares apresentaram sobrepeso/obesidade; a maior prevalência de sobrepeso/obesidade foi observada nas escolas municipais; PAD alterada concentrou-se nas escolas da zona urbana para as meninas e da zona rural leste para os meninos; PAS apresentou-se mais elevada nas escolas da zona urbana e em uma escola da zona rural oeste. Considerações finais: por meio da geolocalização, foi possível identificar os locais que o sobrepeso/obesidade foi mais prevalente; entre estes uma escola da zona rural da região leste, que demonstrou o maior índice de sobrepeso/obesidade. Já, a distribuição da pressãoz arterial alterada demonstrou maiores índices na zona urbana, no sexo feminino e na zona rural no sexo masculino.

Palavras-chave: Obesidade; Sobrepeso; Distribuição espacial da população; Pressão arterial.

\section{ABSTRACT}

Objective: to describe the geographic location of overweight/obesity and abnormal blood pressure of children living in Santa Cruz do Sul, state of Rio Grande do Sul (Brazil). Method: cross-sectional study including 1578 students aged seven to 17 years of 18 local schools. We used the body mass index to evaluate overweight/obesity and systolic blood pressure (SBP) and diastolic blood pressure (DBP) for evaluate blood pressure. The geolocation maps were separated by sex and school and plotted on the map according to the prevalence of variables evaluated. We used the SPSS 20.0 software using descriptive statistics, chi-square test and $t$ test, considering significant differences at $p<0,05$. For the preparation of maps we used Terraview 3.5.O and CoreIDRAW X5 program. Results: the prevalence of overweight/obesity was $27,2 \%$ in boys and $26,5 \%$ in girls. SBP occurred in $14,8 \%$ of boys and $13,6 \%$ of girls. $12,7 \%$ of boys and $12,5 \%$ of girls presented altered DBP. Geolocation pointed to two schools in the rural areas of the east and west regions and one school in the urban zone in the central region of the 
municipality with the highest prevalence of overweight/ obesity, and in the rural school of the eastern region more than $50 \%$ of schoolchildren were overweight/obesity; the worst rates of overweight/obesity were observed in municipal schools; changed DBP concentrated in the urban area schools for girls and the rural east area for boys; SBP was higher in the urban area and in a rural western school. Closing remarks: by means of geolocation it was possible to identify places where overweight/obesity was most prevalent, highlighting a rural school in the eastern region which showed the highest index of overweight/obesity. The distribution of abnormal blood pressure showed higher indices in the urban area for the female sex and in the rural area for the male sex.

Keywords: Obesity; Overweight; Residence Characteristics; Blood pressure.

\section{INTRODUÇÃOO}

A transição nutricional, que muitos países enfrentam, inclusive o Brasil, mostra uma redução nas taxas de desnutrição e aumento do sobrepeso/obesidade. ${ }^{1}$ Este aumento de sobrepeso e obesidade na população constitui um importante problema de saúde pública, principalmente, em crianças e adolescentes. ${ }^{2-4}$

A obesidade é um fator de risco para diabetes tipo 2, diversos tipos de câncer, apneia do sono, asma, doenças psicossociais e, somando-se à dislipidemia e à hipertensão arterial, está associada ao aparecimento de eventos cardiovasculares. ${ }^{5-8}$ Todas estas condições contribuem para um aumento de morbidade e mortalidade prematura, na maioria dos países. ${ }^{9,10}$

Além do aumento crescente desta condição, a hipertensão arterial afeta aproximadamente $5 \%$ das crianças e adolescentes brasileiros. ${ }^{11}$ Estudo, recente no Brasil, demonstrou que a prevalência de hipertensão em adolescentes foi de 9,6\%, sendo a mais elevada, na região Sul do país $(12,5 \%) .^{12}$ Neste sentido, pesquisa internacional revelou que, na faixa etária de oito a 17 anos, existe uma prevalência de pré-hipertensão e hipertensão de $10 \%$ e $4 \%$, respectivamente, sendo que a hipertensão aumenta proporcionalmente, à medida que aumenta a obesidade infantil. ${ }^{13}$

O mapeamento de doenças é de suma importância, quando se quer uma vigilância sobre esta, pois o conhecimento da distribuição espacial da área, onde esta ocorre, possibilita determinar padrões da situação de saúde, evidenciando disparidades geográficas. A delimitação das áreas de risco tem contribuído para fundamentar melhores decisões de planejamento e de gestão de políticas públicas. ${ }^{14,15}$

Este mapeamento pode ser realizado por meio da geolocalização, definida como um conjunto de tecnologias de coleta, tratamento, manipulação e apresentação de informações espaciais. ${ }^{14} \mathrm{O}$ cruzamento de dados, realizados pela sua técnica, facilita a inserção dos dados intersetoriais e acaba expressando as relações entre as condições de vida e os níveis de saúde e doença. ${ }^{16}$ Dessa maneira, a geolocalização é uma ferramenta de suma importância, na área da saúde pública, uma vez que torna possível o reconhecimento da realidade local, possibilitando aos gestores públicos a reorientação das ações em saúde. ${ }^{15,16}$

Devido à importância da geolocalização na área de vigilância em saúde e o aumento crescente de sobrepeso/obesidade e hipertensão arterial, o presente estudo objetiva descrever a geolocalização de casos de sobrepeso/obesidade e pressão arterial alterada de escolares residentes em Santa Cruz do Sul/RS.

\section{MÉTODO}

Foi realizado um estudo transversal, incluindo 1.578 escolares, de 18 escolas da área urbana e rural do município de Santa Cruz do Sul, no Estado do Rio Grande do Sul. Esta pesquisa foi realizada durante o ano de 2008, com escolares de sete a 17 anos, selecionados aleatoriamente de uma amostra estratificada por conglomerados (centro, norte, sul, leste e oeste). Destas escolas, duas são particulares, seis municipais, sendo duas da zona rural (sul e oeste) e 10 escolas estaduais, sendo duas da zona rural (norte e leste).

O projeto de pesquisa foi encaminhado e aprovado pelo Comitê de Ética em Pesquisa da Universidade de Santa Cruz do Sul, sob o protocolo $n^{\circ} 4.913-2007$, em conformidade com a Declaração de Helsinki. Todos os escolares que participaram tiveram o Termo de Consentimento Livre e Esclarecido, devidamente assinado pelos pais ou responsáveis.

O índice de massa corporal (IMC) foi calculado pela razão do peso $(\mathrm{kg}) /$ altura $^{2}(\mathrm{~m})$ e posteriormente classificado de acordo com o protocolo de Conde e Monteiro. ${ }^{17}$ A pressão arterial (PA) foi aferida duas vezes, sempre pelo mesmo avaliador, com o aluno sentado, em repouso. Utilizaram-se esfigmomanômetro e estetoscópio no braço esquerdo e manguito adequado para o perímetro braquial do aluno. A pressão foi classificada por meio dos percentis 90 e 95, para a faixa limítrofe e hipertensão, respectivamente, conforme parâmetros da V Diretriz Brasileira de Hipertensão Arterial, da Sociedade Brasileira de Hipertensão/SBC. ${ }^{18}$ A faixa etária foi definida através do Estatuto da Criança e do Adolescente, que define criança a pessoa com até doze anos incompletos e adolescentes entre doze e dezoito anos de idade. ${ }^{19}$

Para a confecção dos mapas, a análise dos resultados foi realizada por escola e por sexo, identificando o percentual de indivíduos com sobrepeso/obesidade e pressão arterial alterada; em seguida, os resultados foram transferidos para o programa Terraview 3.5.0, onde se realizou a construção do mapa da cidade. Após, utilizou-se o programa CoreIDRAW X5, para realizar a marcação dos pontos em cada escola.

Para análise dos dados utilizou-se o programa SPSS 20.0 for Windows (IBM Corp. Released 2011, Armonk, NY: IBM Corp.USA) e empregou-se a estatística descritiva. Utilizou-se o teste de qui-quadrado e teste $t$, considerando diferenças significativas, para $p<0,05$, com um intervalo de confiança de $95 \%$.

\section{RESULTADOS}

De acordo com a tabela 1, observa-se que $53 \%$ dos escolares são do sexo masculino, $56,9 \%$ são 
adolescentes, $88,8 \%$ estudam em escolas públicas e $77,8 \%$ são moradores da zona urbana. Em relação às características antropométricas dos escolares, 27,2\% dos meninos e $26,5 \%$ das meninas apresentam excesso de peso (sobrepeso e obesidade). O percentual de escolares com pressão arterial alterada (limítrofes e hipertensos) é de $14,8 \%$ entre os meninos e $13,6 \%$ entre as meninas, para pressão arterial sistólica (PAS); para pressão arterial diastólica (PAD), o percentual encontrado foi de $12,7 \%$ e $12,5 \%$, para meninos e meninas, respectivamente. Houve diferença significativa entre meninos e meninas para o IMC $(p=001)$ e pressão arterial sistólica $(p=0,009)$.

$\mathrm{Na}$ tabela 2, são apresentados os dados dos sujeitos estratificados por sexo e classificação do IMC (G1 = Baixo Peso/Normal e G2= Sobrepeso/Obesidade). Entre os indivíduos do sexo masculino, com sobrepeso/obesidade, $72,7 \%$ são residentes da zona urbana

Tabela 1 - Características demográficas, antropométricas e pressóricas.

\begin{tabular}{|c|c|c|c|c|}
\hline Variáveis & Masculino N (\%) & Feminino $\mathbf{N}(\%)$ & $\mathbf{p}$ & $N(\%)$ \\
\hline Sexo & $836(53,0)$ & $742(47,0)$ & - & $1578(100)$ \\
\hline \multicolumn{5}{|l|}{ Faixa etária } \\
\hline Criança (7-11 anos) & $362(43,3)$ & $318(42,9)$ & 0,450 & $680(43,1)$ \\
\hline Adolescente (12-17 anos) & $474(56,7)$ & $424(57,1)$ & & $898(56,9)$ \\
\hline \multicolumn{5}{|l|}{ Tipo de escola } \\
\hline Pública & $739(88,4)$ & $662(89,2)$ & 0,332 & $1401(88,8)$ \\
\hline Privada & $97(11,6)$ & $80(10,8)$ & & $177(11,2)$ \\
\hline \multicolumn{5}{|l|}{ Região de moradia } \\
\hline Zona urbana & $648(77,5)$ & $580(78,2)$ & 0,401 & $1228(77,8)$ \\
\hline Zona rural & $188(22,5)$ & $162(21,8)$ & & $350(22,2)$ \\
\hline \multicolumn{5}{|l|}{ IMC } \\
\hline Baixo peso & $7(0,8)$ & $18(2,5)$ & 0,001 & $25(1,6)$ \\
\hline Normal & $602(72,0)$ & $527(71,0)$ & & $1129(71,5)$ \\
\hline Sobrepeso & $177(21,2)$ & $127(17,1)$ & & $304(19,3)$ \\
\hline Obesidade & $50(6,0)$ & $70(9,4)$ & & $120(7,6)$ \\
\hline \multicolumn{5}{|l|}{ PAS } \\
\hline Normotenso & $712(85,2)$ & $641(86,4)$ & 0,009 & $1353(85,7)$ \\
\hline Limítrofe & $35(4,2)$ & $48(6,5)$ & & $83(5,3)$ \\
\hline Hipertenso & $89(10,6)$ & $53(7,1)$ & & $142(9,0)$ \\
\hline \multicolumn{5}{|l|}{ PAD } \\
\hline Normotenso & $730(87,3)$ & $649(87,5)$ & 0,993 & $1379(87,4)$ \\
\hline Limítrofe & $61(7,3)$ & $53(7,1)$ & & $114(7,2)$ \\
\hline Hipertenso & $45(5,4)$ & $40(5,4)$ & & $85(5,4)$ \\
\hline Variáveis & Masculino $\bar{\chi}$ (DP) & Masculino $\bar{\chi}$ (DP) & p & Total $\bar{\chi}$ (DP) \\
\hline Idade & $12,01(2,95)$ & $11,99(2,92)$ & 0,897 & $12,00(2,93)$ \\
\hline IMC (kg/m2) & $19,32(3,75)$ & $19,25(3,82)$ & 0,707 & $19,29(3,78)$ \\
\hline PAS (mmHg) & $105,78(16,14)$ & $103,20(14,45)$ & 0,001 & $104,56(15,42)$ \\
\hline PAD (mmHg) & $63,80(10,95)$ & $62,65(11,16)$ & 0,038 & $63,26(11,06)$ \\
\hline
\end{tabular}

* $\bar{\chi}$ (Desvio Padrão); IMC: Índice de Massa Corporal; PAS: Pressão arterial sistólica; PAD: Pressão arterial diastólica. Valores significativos para $p<0,05$.

Tabela 2 - Classificação das variáveis demográficas e pressóricas de acordo com o estado nutricional.

\begin{tabular}{|c|c|c|c|c|c|c|}
\hline \multirow[b]{2}{*}{ Faixa etária } & \multicolumn{2}{|c|}{ Masculino } & \multirow[t]{2}{*}{$\mathbf{p}$} & \multicolumn{2}{|c|}{ Feminino } & \multirow[t]{2}{*}{$\mathbf{p}$} \\
\hline & Grupo 1 N (\%) & Grupo 2 N (\%) & & Grupo 1 N (\%) & Grupo 2 N (\%) & \\
\hline Adolescente (12-17 anos) & $253(41,5)$ & $109(48,0)$ & 0,100 & $211(38,7)$ & $107(54,3)$ & $<0,001$ \\
\hline $\begin{array}{l}\text { Tipo de escola } \\
\text { Pública }\end{array}$ & $356(58,5)$ & $118(52,0)$ & & $334(61,3)$ & $90(45,7)$ & \\
\hline Privada & $534(87,7)$ & $205(90,3)$ & 0,332 & $482(88,4)$ & $180(91,4)$ & 0,286 \\
\hline $\begin{array}{l}\text { Região da escola } \\
\text { Zona Urbana }\end{array}$ & $75(12,3)$ & $22(9,7)$ & & $63(11,6)$ & $17(8,6)$ & \\
\hline Zona rural & $483(79,3)$ & $165(72,7)$ & 0,050 & $431(79,1)$ & $149(75,6)$ & 0,316 \\
\hline $\begin{array}{l}\text { PAS }(\mathrm{mmHg}) \\
\text { Normotenso }\end{array}$ & $126(20,7)$ & $62(27,3)$ & & $114(20,9)$ & $48(24,4)$ & \\
\hline $\begin{array}{l}\text { Limítrofe } \\
\text { Hipertenso }\end{array}$ & $\begin{array}{c}548(90,0) \\
20(3,3)\end{array}$ & $\begin{array}{c}164(72,2) \\
15(6,7)\end{array}$ & $<0,001$ & $\begin{array}{c}489(89,7) \\
26(4,8)\end{array}$ & $\begin{array}{c}152(77,1) \\
22(11,2)\end{array}$ & $<0,001$ \\
\hline $\begin{array}{l}\text { PAD (mmHg) } \\
\text { Normotenso }\end{array}$ & $41(6,7)$ & $48(21,1)$ & & $30(5,5)$ & $23(11,7)$ & \\
\hline $\begin{array}{l}\text { Limítrofe } \\
\text { Hipertenso }\end{array}$ & $\begin{array}{c}547(89,8) \\
41(6,7) \\
21(3,5)\end{array}$ & $\begin{array}{c}183(80,6) \\
20(8,8) \\
24(10,6)\end{array}$ & $<0,001$ & $\begin{array}{c}491(90,1) \\
38(7,0) \\
16(2,9)\end{array}$ & $\begin{array}{c}158(80,2) \\
15(7,6) \\
24(12,2)\end{array}$ & $<0,001$ \\
\hline
\end{tabular}

Grupo 1: Baixo peso/normal; Grupo 2: Sobrepeso/obesidade; PAS: Pressão arterial sistólica; PAD: Pressão arterial diastólica. Valores significativos para p<0,05 
$(p=0,050)$ e $52,0 \%$ são adolescentes $(p=0,100)$; a PAS alterada (limítrofe e hipertenso) esteve presente em $27,8 \%(p<0,001)$ e PAD alterada em 19,4\% ( $<<0,001)$. Entre os indivíduos com sobrepeso/obesidade, no sexo feminino, 91,4\% são pertencentes a escolas públicas, $75,6 \%$ são moradores da zona urbana e $54,3 \%$ são crianças $(p<0,001)$; PAS alterada (limítrofe e hipertenso) esteve presente em 22,9\% (p<0,001) e PAD alterada, em 19,8\% ( $p<0,001)$. Destaca-se que, para ambos os sexos, os índices de PAS e PAD alteradas foram maiores nos sujeitos com sobrepeso/obesidade, quando comparados a seus pares eutróficos.

Através da geolocalização dos casos de sobrepeso/obesidade nas escolas (Figura 1), observou-se que, a maior prevalência de sobrepeso/obesidade encontra-se em uma escola da zona rural na região leste (51-60/100) para ambos os sexos, seguido de uma escola da zona urbana na região central (41-50/100 no sexo feminino e $31-50 / 100$ no sexo masculino) e uma escola rural da região oeste (31-50/100), para ambos os sexos. Destaca-se que a primeira escola indicada não possuía cantina, bem como acompanhamento e orientação nutricional. Em relação ao tipo de escola, a maior prevalência de sobrepeso/obesidade foi observada nas escolas municipais.

A distribuição da pressão arterial alterada por sexo e por escola está ilustrada na figura 2 ( $A$ e B, para PAS e PAD, respectivamente); observa-se que a PAS, de modo geral, apresentou-se mais elevada na zona urbana, identificada no centro do mapa (21-30/100), bem como em uma escola da zona rural na região oeste, principalmente no sexo masculino (21-30/100). A PAD também foi mais elevada na zona urbana no sexo feminino (51-60/100), enquanto que no sexo masculino, o índice mais elevado encontra-se na região leste da zona rural (31-40/100).

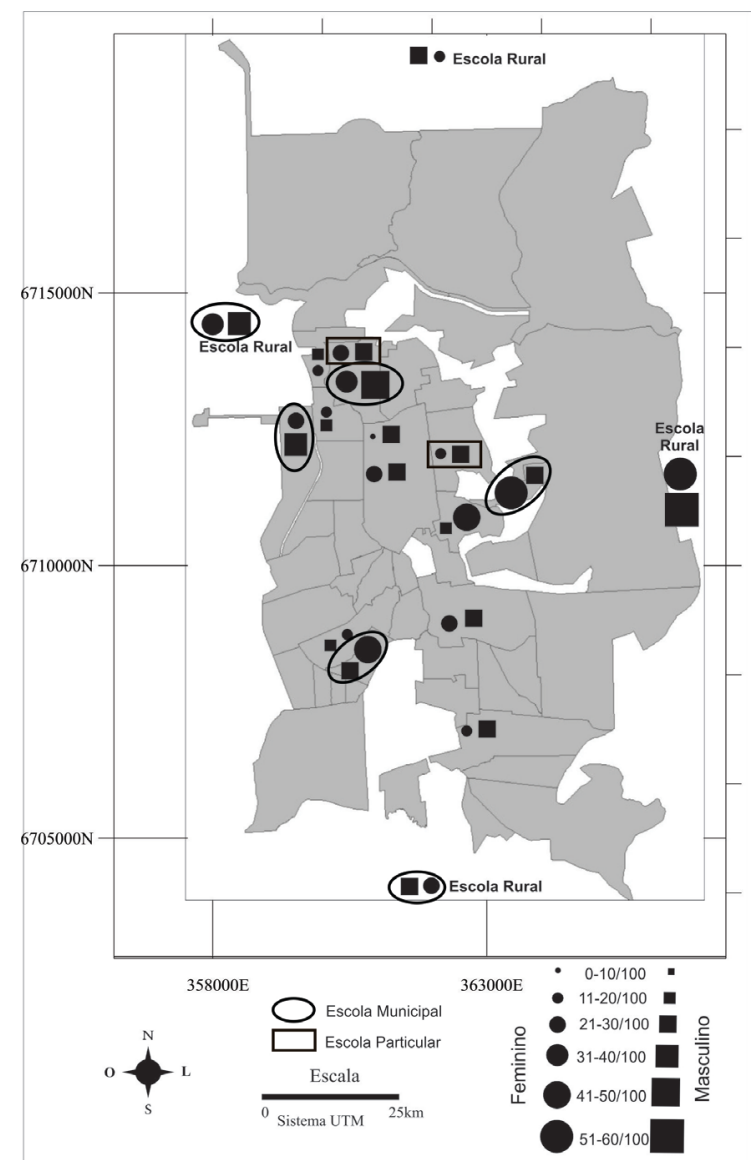

Figura 1 - Mapa do município de Santa Cruz do Sul indicando a prevalência de sobrepeso e obesidade em escolares do município, estratificado por sexo.
(A)

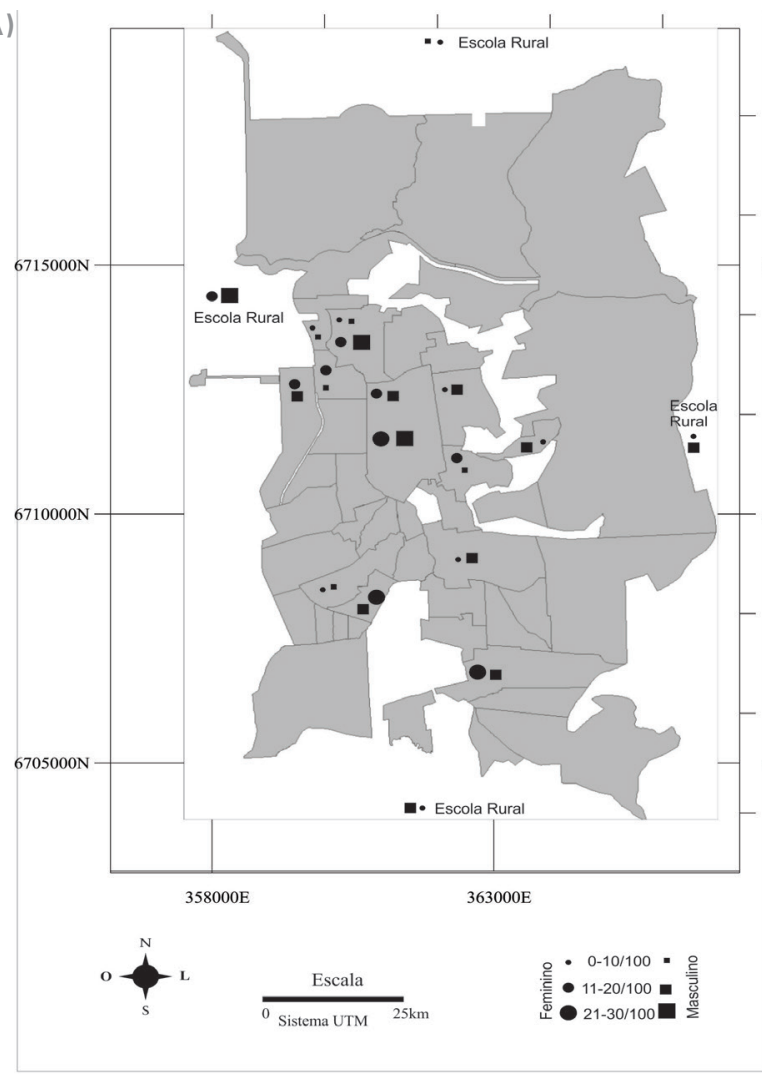

(B)

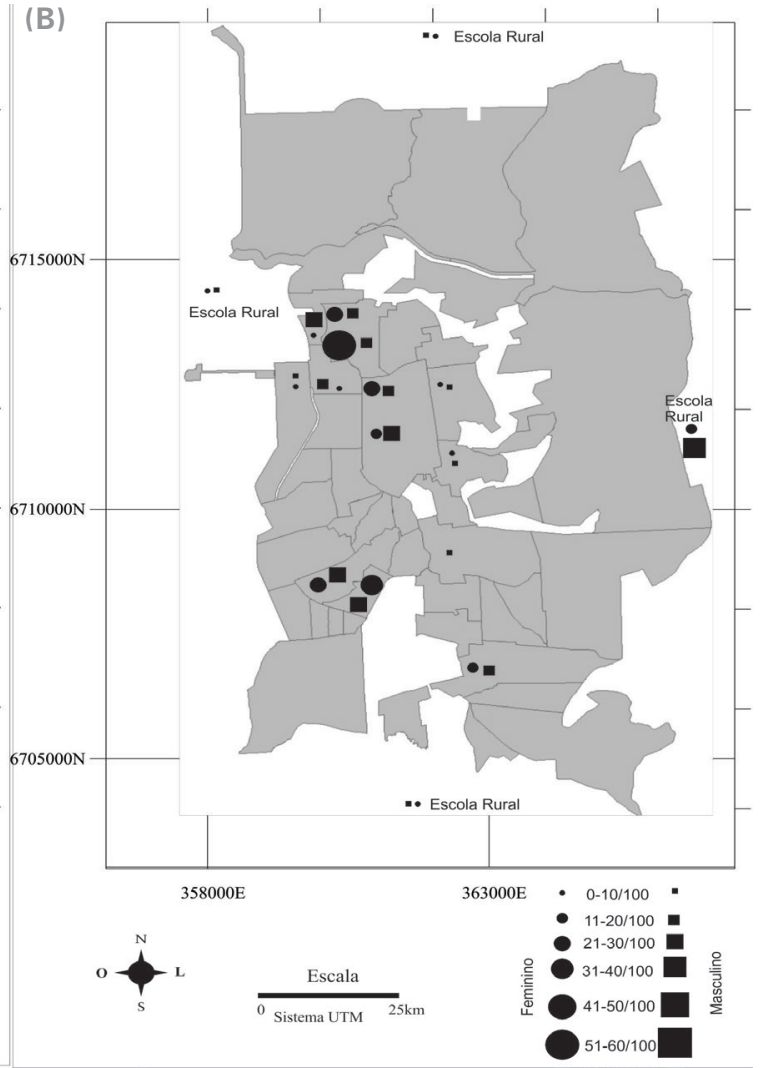

Figura 2 - Mapas do município de Santa Cruz do Sul indicando em (A) PAS e (B) PAD em estudantes de 18 escolas localizadas no município. 


\section{DISCUSSÃO}

Em nosso estudo, o sobrepeso e obesidade estiveram presentes em $26,9 \%$ dos escolares, sendo mais prevalentes no sexo masculino. Estudo longitudinal realizado anteriormente na cidade de Santa Cruz do Sul-RS, com uma amostra de 414 escolares de sete a 17 anos, evidenciou um aumento no índice de sobrepeso/obesidade de $18,08 \%$ em 2005 para $20,43 \%$ em 2008. ${ }^{20}$ Diante destes resultados, observa-se que o índice de excesso de peso vem aumentando na cidade estudada, o que indica que medidas preventivas e educativas devem ser adotadas de forma urgente, de modo a trabalhar com estes escolares, para tratar suas situações no intuito de reduzir estes índices. Do mesmo modo, observa-se que em Shandong, na China, em estudo com 72.755 escolares de sete a 18 anos, os índices de obesidade também se elevaram, após um período de quatro anos, havendo um aumento de $16,63 \%$ para $20,37 \%$ em meninos, e $8,13 \%$ para $11,61 \%$ nas meninas. ${ }^{21}$

Quanto à prevalência de sobrepeso e obesidade, este foi menor em estudo de Keane et al. ${ }^{22}$ em Cork, Irlanda, em que, em amostra de 826 escolares de oito a 11 anos, verificou-se índice de $23,7 \%$ de sobrepeso/ obesidade. Já, em Dehradun, Uttaranchal, em estudo com 1266 escolares, os níveis foram consideravelmente menores, em que $15,6 \%$ apresentaram sobrepeso, dos quais $5,4 \%$ eram obesos. ${ }^{23}$ Diferentemente, níveis mais altos aos nossos foram verificados em Barcelona na Espanha, em que a prevalência de sobrepeso foi de $24,0 \%$ e de obesidade $12,7 \%$, em uma amostra representativa de 3262 escolares de oito a nove anos, sendo essa última mais comum em meninos. ${ }^{24}$

Em Caxemira na Índia, um estudo com maior representação de escolares da rede privada de ensino, verificou $6,69 \%$ de sobrepeso e $4,64 \%$ de obesidade, em 2024 escolares de seis a 18 anos. ${ }^{25}$ Esses valores são expressivamente menores aos encontrados em nosso estudo, sendo que nossa amostra foi representada em maior parte $(88 \%)$ pela rede pública de ensino.

De forma específica, o sobrepeso/obesidade afetou $27,2 \%$ dos meninos e $26,5 \%$ das meninas, no presente estudo. Estes dados são semelhantes aos achados em Pelotas-RS, em que $26,4 \%$ dos meninos e $25,3 \%$ das meninas também apresentaram sobrepeso/ obesidade. ${ }^{26}$ Isoladamente, a prevalência de sobrepeso em nosso estudo foi maior entre os meninos (21,2\%) e a obesidade entre as meninas $(9,4 \%)$. Mazaro et al. ${ }^{27}$ verificaram que ambos (sobrepeso e obesidade) estavam mais prevalentes no sexo masculino em estudo em Sorocaba-SP.

Em nosso estudo, o sobrepeso/obesidade esteve mais presente nos adolescentes do sexo masculino $(52,0 \%)$ e nas crianças do sexo feminino $(54,3 \%)$. Pesquisa realizada por Oliveira, Cerqueira e Oliveira, ${ }^{28}$ em Feira de Santana-BA, demonstra que a obesidade infantil e adulta está mais prevalente no sexo feminino, não havendo motivos definidos para sua ocorrência. Estudo realizado por Balaban e Silva ${ }^{29}$ em Recife-PE, também verificou o sobrepeso e obesidade mais frequente em crianças, do que adolescentes. Os autores explicam que um moderado excesso de peso em crianças poderá ser compensado no futuro pelo crescimento. Entretanto, na adolescência (12 a 17 anos), o maior percentual de sobrepeso/obesida- de foi encontrado no sexo masculino (24,9\%).

No presente estudo, os sujeitos com sobrepeso/ obesidade apresentaram maiores índices de PAS e PAD alteradas, quando comparados a seus pares eutróficos, em ambos os sexos, sendo que os meninos obtiveram os maiores percentuais. Do mesmo modo, estudo realizado em Montenegro, país do sudeste da Europa, com 3254 crianças e adolescentes de sete a 13 anos, verificou que os escolares com obesidade tinham $68 \%$ maior probabilidade de apresentar hipertensão arterial. ${ }^{30}$ Concomitantemente, Costanzi et al. ${ }^{31}$ também verificou proporção maior de pressão arterial alta para crianças obesas e/ ou com sobrepeso, em comparação com crianças de peso normal, em uma amostra de 1413 escolares de sete a 12 anos de Caxias-RS. Manios et al., ${ }^{32}$ em estudo com quatro municípios da Grécia, também verificaram maior prevalência de hipertensão arterial em crianças e adolescentes obesos, bem como Kolpa et al., ${ }^{33}$ em Tarnow na Polônia, observaram que a proporção de PA alterada aumentava, conforme o índice de acúmulo de gordura corporal também se elevava.

O percentual de escolares com PAS alterada é de $14,8 \%$ entre os meninos e $13,6 \%$ entre as meninas e com PAD alterada é de $12,7 \%$ e $12,5 \%$, para meninos e meninas, respectivamente, em nosso estudo. Estudo realizado na Grécia, com 2.571 escolares de nove a 13 anos, verificou pré-hipertensão em $14,2 \%$ e hipertensão em 23,0\%, sendo que as meninas apresentaram índices maiores de PA elevada, diferente do encontrado em nosso estudo. O mesmo estudo demonstrou hipertensão sistólica $(11,9 \%)$ mais prevalente que a hipertensão diastólica $(4,5 \%)$, sendo maior nos meninos, corroborando com os achados do presente estudo. ${ }^{34}$ Diferentemente, na Inglaterra, um estudo, com 7.329 escolares de 10 a 16 anos de idade demonstrou valores mais expressivos de PAD alterada, ao invés de PAS, $44 \%$ e $23 \%$ respectivamente. ${ }^{35}$

Outros estudos demonstraram índices menores de pressão arterial elevada. Em Banguecoque, na Tailândia, verificou-se pré-hipertensão de 5,7\% e 2,7\% para meninos e meninas respectivamente e hipertensão de $4,7 \%$ para meninos e 3,2\% para meninas, em uma amostra de 693 escolares de oito a 12 anos. ${ }^{36} \mathrm{Em} \mathrm{Lu}$ anda, Angola, foi verificada pressão arterial elevada em $14,6 \%$ dos escolares de sete a 11 anos, havendo pré-hipertensão em $10,4 \%$ dos meninos e $9,9 \%$ das meninas, e hipertensão em $5,2 \%$ e $4,1 \%$ dos meninos e meninas respectivamente. $\mathrm{O}$ mesmo estudo também verificou associação entre o acúmulo de gordura corporal e o aumento da pressão arterial, reafirmando o achado de nosso estudo. ${ }^{37}$ Já, Rinaldi et al. ${ }^{38}$ identificaram 12,0\% dos escolares limítrofes e hipertensos em Botucatu-SP, resultados estes semelhantes aos do presente estudo.

De acordo com o presente estudo, entre os escolares que estudavam em escolas da zona urbana, 25,6\% tinham sobrepeso/obesidade, enquanto que, entre os escolares da zona rural, a frequência de obesidade foi de $31,4 \%$. Em pesquisa realizada por Guimarães et al. ${ }^{39}$ em Florianópolis-SC, observou-se que na zona urbana o IMC foi significativamente maior pelo fato dos escolares terem maior acesso à alimentação industrializada/fast food e alimentos altamente calóricos.

Porém, o sobrepeso e obesidade são determinados por múltiplos fatores, dentre eles, tem se destacado, a 
falta de atividade física regular. ${ }^{40}$ Quando considerado que crianças mais ativas apresentam índice de massa corporal e percentual de gordura menos elevado, pode-se levar em conta, a atividade física como um fator protetor contra sobrepeso e obesidade. ${ }^{41} \mathrm{~A}$ implementação de exercícios controlados e regulares, pode ser útil em escolas para melhora da aptidão física dos escolares. Entretanto, apesar dos benefícios da atividade física escolar, na maioria das escolas entrevistadas por esta pesquisa, ficou claro que existem poucas sessões semanais de Educação Física (via de regra uma ou duas sessões semanais), principalmente nas escolas públicas.

Quanto à verificação da prevalência de sobrepeso e obesidade em crianças e adolescentes por meio de um mapa epidemiológico, estudo realizado na Espanha identificou prevalência maior na extremidade sul do país; além disso, mostrou-se superior em áreas urbanas e em municípios em que a posição socioeconômica é mais baixa. ${ }^{42}$ Já, na Suécia, o sobrepeso/obesidade é mais prevalente em áreas rurais $(22,7 \%)$ e em locais com baixo nível educacional (25,5\%). ${ }^{43} \mathrm{Na}$ Islândia, mapas demonstram que há variação geográfica de sobrepeso/obesidade nos adolescentes avaliados, sendo mais prevalente em áreas rurais. ${ }^{44}$

\section{CONSIDERAÇÕES FINAIS}

Por meio da geolocalização, foi possível identificar os locais em que o sobrepeso/obesidade foi mais prevalente, sendo este em duas escolas da zona rural das regiões leste e oeste e uma escola da zona urbana na região central do município. Do mesmo modo, a maior prevalência de sobrepeso/obesidade foi observada nas escolas municipais. Quanto à pressão arterial, a mesma mostrou-se mais alterada em escolares com sobrepeso/ obesidade, principalmente, a PAS para os meninos. A distribuição por geolocalização da pressão arterial alterada também demonstrou maior prevalência na zona urbana no sexo feminino e na zona rural no sexo masculino.

Deste modo, o diagnóstico da prevalência de obesidade/sobrepeso, PAS e PAD alterada e o uso da geolocalização foi de vital importância para detectar alterações e mapear as áreas consideradas de maior risco, ou seja, aquelas escolas em que devem ser realizadas intervenções em saúde. Essa identificação é essencial na população estudada, pois a tendência de sobrepeso/obesidade vem aumentando nessa faixa etária, sendo de suma importância a identificação dos mesmos para posterior desenvolvimento de projetos e ações específicas.

\section{REFERÊNCIAS}

1. WHO. Reducing Risks, Promoting Healthy Life. Geneva, 2002; p. 01-14.

2. Cintra IP, Passos MA, Fisberg M, Machado HC. Evolution of body mass index in two historical series of adolescents. J Pediatr 2007;83(2):157-62. doi: 10.1590/S0021-75572007000200010

3. Lamounier JA, Abrantes MM, Colosimo, EA. Prevalência de sobrepeso e obesidade em crianças e adolescentes das regiões Sudeste e Nordeste. J Pediatr 2002;78(4):335-40. doi: 10.1590/S0021-75572002000400014
4. Sahoo K, Sahoo B, Choudhury AK, Sofi NY, Kumar R, Bhadoria AS. Childhood obesity: causes and consequences. J Fam Med Prim Care 2015;4(2):187-92. doi: 10.4103/22494863.154628

5. Dirisamer A, Widhalm K. Lipoprotein (a) as a potent risk indicator for early cardiovascular disease. Acta Paediatr 2002;91(12):1313-7. doi: 10.1111/j.1651-2227.2002. tb02826.x

6. Wiegman A, Rodenburg J, De Jongh S, Defesche JC, Bakker, HD, Kastelein JJ, Sijbrands EJ. Family history and cardiovascular risk in familial hypercholesterolemia: data in more than 1000 children. Circulation 2003; 25;107(11):14738. doi: 10.1161/01.CIR.0000058166.99182.54

7. World Cancer Research Fund/American Institute For Cancer Research. Food, Nutrition, Physical Activity, and the Prevention of Cancer: a Global Perspective. Washington DC: AICR, 2007.

8. Aceves-Martins M, Llauradó E, Tarro L, Solà R, Giralt M. Obesity-promoting factors in Mexican children and adolescents: challenges and opportunities. Glob Health Action. 2016; 9. doi: 10.3402/gha.v9.29625

9. Mello ED, Luft VC, Meyer F. Childhood obesity Towards effectiveness. J Pediatr 2004;80(3):173-82. doi: 10.2223/ JPED.1180

10. Teixeira PDS, Reis BZ, Vieira DAS, Costa D, Costa JO, Raposo OFF, Wartha ERSA, Netto RSM. Intervenção nutricional educativa como ferramenta eficaz para mudança de hábitos alimentares e peso corporal entre praticantes de atividade física. Ciênc saúde coletiva 2013;18(2):347-56. doi: 10.1590/S1413-81232013000200006

11. Sociedade Brasileira De Hipertensão. Acesso em $>10 \mathrm{de}$ abril de 2017. Disponível em: http://www.sbh.org.br/geral/ oque-e-hipertensao.asp.

12. Bloch $\mathrm{KV}$, Klien $\mathrm{CH}$, Szklo $\mathrm{M}$, et. al. ERICA: prevalences of hypertension and obesity in Brazilian adolescentes. Rev Saúde Pública 2016;50(1):9s. doi: 10.1590/s015188787.2016050006685

13. Din-Dzietham R, Liu Y, Bielo MV, Shamsa F. High Blood Pressure Trends in Children and Adolescents in National Surveys, 1963 to 2002. Circulation 2007;116(13):1488-96. doi: 10.1161/CIRCULATIONAHA.106.683243

14. Pina MF, Santos SM. Potencialidades dos Sistemas de Informações Geográficas na Área da Saúde. In: Najar AL, Marques EC. Saúde e Espaço: Estudos metodológicos e técnicas de análise. Rio de Janeiro. FIOCRUZ. 1998.

15. Lopes VBDB, Silva E, Volc SM, Sefrin SRG, Peruchi JAM. Utilização do Sistema de Informação Geográfica para Análise do Câncer em Santa Catarina. Hygeia 2015;11(21):153-73.

16. Grow HMG, Cook AJ, Arterburn DE, Saelens BE, Drewnowski A, Lozano P. Child obesity associated with social disadvantage of children's Neighborhoods. Soc Sci Med 2010;71(3):58491. doi: 10.1016/j.socscimed.2010.04.018

17. Conde WL, Monteiro CA. Body mass index cutoff points for evaluation of nutritional status in Brazilian children and adolescents. J Pediatr 2006;82(4):266-72. doi: 10.2223/ JPED.1502

18. SBC/SBH/SBN. Sociedade Brasileira de Cardiologia/ Sociedade Brasileira de Hipertensão/Sociedade Brasileira de Nefrologia. V Diretrizes Brasileiras de Hipertensão Arterial. São Paulo: SBC/SBH/SBN, 2006.

19. BRASIL. Estatuto da criança e do adolescente (1990). Estatuto da criança e do adolescente. - 7.ed. - Brasília: Câmara dos Deputados, Edições Câmara, 2010. 
20. Reuter EM, Reuter CP, Burgos LT, Miriam Beatris Reckziegel MB, Nedel FB, Albuquerque IM, Pohl HH, Burgos MS. Obesity and arterial hypertension in schoolchildren from Santa Cruz do Sul - RS, Brazil. Rev Assoc Med Bras 2012;58(6):666672. doi:10.1590/S0104-42302012000600010

21. Zhang $Y X$, Wang SR, Chen M, Cheng Y. Recent Trends in Body Mass Index and Waist Circumference among Children and Adolescents in Shandong China. J Trop Pediatr. 2017. doi: 10.1093/tropej/fmx013.

22. Keane E, Li X, Harrington JM, Fitzgerald AP, Perry IJ, Kearney PM. Physical Activity, Sedentary Behaviour and the Risk of Overweight and Obesity in School Aged Children. Pediatr Exerc Sci 2017;7:1-27. doi: 10.1123/pes.2016-0234

23. Bhargava M, Kandpal SD, Aggarwal P. Physical activity correlates of overweight and obesity in school-going children of Dehradun, Uttarakhand. J Family Med Prim Care 2016;5(3):564-568. doi: 10.4103/2249-4863.197281

24. Sánchez-Martínez F, Torres Capcha P, Serral Cano G, Valmayor Safont S, Castell Abat C, Ariza Cardenal C. Factors Associated with Overweight and Obesity in Schoolchildren from 8 to 9 Years Old. Barcelona, Spain. Rev Esp Salud Publica 2016;90:e1-e11.

25. Ganie MA, Bhat GA, Wani IA, Rashid A, Zargar SA, Charoo BA, Shah ZA, Mudassar S. Prevalence, risk factors and consequences of overweight and obesity among schoolchildren: a cross-sectional study in Kashmir, India. J Pediatr Endocrinol Metab 2017;30(2):203-209. doi: 10.1515/ jpem-2016-0133

26. Terres NG, Pinheiro RT, Horta BL, Pinheiro KAT, Horta LL. Prevalence and factors associated to overweight and obesity in adolescents. Rev Saúde Pública 2006;40(4):627-33. doi: 10.1590/S0034-89102006000500011

27. Mazaro IAR, Zanolli ML, Antonio MARGM, Morcillo AM, Zambon MP. Obesity and cardiovascular risk factors in school children from Sorocaba, SP. Rev Assoc Med Bras 2011;57(6):674-80. doi: 10.1590/S0104 42302011000600015

28. Ronque VER, Cyrino ES, Dórea VR, Serassuelo JR, Galdi EHG, Arruda M. Prevalência de sobrepeso e obesidade em escolares de alto nível socioeconômico em Londrina, Paraná, Brasil. Rev Nutr 2005;18(6)709-17. doi: 10.1590/S141552732005000600001

29. Baruki SBS, Rosado LEFPL, Rosado GP, Ribeiro RCL. Association between nutritional status and physical activity in Municipal Schools in Corumbá - MS. Rev Bras Med Esporte 2006;12(2):90-4. doi: 10.1590/S1517 86922006000200007

30. Martinovic M, Belojevic G, Evans GW, Kavaric N, Asanin B, Pantovic S, Jaksic M, Boljevic J. Hypertension and correlates among Montenegrin schoolchildren-a crosssectional study. Public Health 2017;147:15-19. doi: 10.1016/j. puhe.2017.02.007

31. Costanzi CB, Halpern R, Rech RR, Bergmann ML, Alli LR, Mattos AP. Associated factors in high blood pressure among schoolchildren in a middle size city, southern Brazil. J Pediatr 85(4):335-40. doi:10.2223/JPED.1913.

32. Manios $Y$, Karatzi K, Protogerou AD, Moschonis G, Tsirimiagou C, Androutsos O, Lionis C, Chrousos GP. Prevalence of childhood hypertension and hypertension phenotypes by weight status and waist circumference: the Healthy Growth Study. Eur J Nutr. 2017. doi: 10.1007/s00394-017-1398-y

33. Kolpa M, Jankowicz-Szymanska A, Jurkiewicz B. High-Normal Arterial Blood Pressure in Children With Excess Body Weight. Iran J Pediatr 2016;26(4):e4677. doi: 10.5812/ijp.4677

34. Karatzi K, Protogerou AD, Moschonis G, Tsirimiagou C, Androutsos O, Chrousos GP, Lionis C, Manios Y. Prevalence of hypertension and hypertension phenotypes by age and gender among schoolchildren in Greece: The Healthy Growth Study. Atherosclerosis 2017;259:128-133. doi: 10.1016/j.atherosclerosis.2017.01.027

35. Cohen DD, López-Jaramillo P, Fernández-Santos JR, CastroPiñero J, Sandercock G. Muscle strength is associated with lower diastolic blood pressure in schoolchildren. Prev Med 2017;95:1-6. doi: 10.1016/j.ypmed.2016.11.006

36. Sukhonthachit $\mathrm{P}$, Aekplakorn W, Hudthagosol C, Sirikulchayanonta $\mathrm{C} 1$. The association between obesity and blood pressure in Thai public school children. BMC Public Health 2014;14:729. doi: 10.1186/1471-2458-14-729

37. Silva $A B$, Capingana $D P$, Magalhães $P$, Gonçalves $M A$, Molina MD, Rodrigues SL, Baldo MP, Mateus MS, Mill JG. Cardiovascular risk factors in pre-pubertal schoolchildren in Angola. Cardiovasc J Afr 2016;27(5):315-321. doi: 10.5830/ CVJA-2016-029

38. Rinaldi AEM, Nogueira PCK, Riyuso MC, Olbrich-Neto J, Gabriel GFCP, Macedo CS, Burini RC. Prevalence of elevated blood pressure in children and adolescents attending highschool. Rev Paul Pediatr 2012;30(1):79-86. doi: 10.1590/ S0103-05822012000100012

39. Guimarães ACA, Feijó I, Soares A, Fernandes S, Machado Z, Parcias SR. xcesso de peso e obesidade em escolares: associação com fatores biopsicológicos, socioeconômicos e comportamentais. Arq Bras Endocrinol Metabol 2012;56(2):142-8. doi: 10.1590/S000427302012000200008

40. Oliveira AMA, Cerqueira EMM, Oliveira AC. Prevalência de Sobrepeso e Obesidade Infantil em população urbana: detecção pela família $\mathrm{x}$ diagnóstico médico. J Pediatr 2003;79(4):325-8.

41. Balaban G, Silva GAP. Prevalência de sobrepeso e obesidade em crianças e adolescentes de uma escola da rede privada de Recife. J Pediatr 2001;77(2):96-00. doi 10.1590/S002175572001000200008

42. Pizarro JV, Royo-Bordonada MA. Prevalence of childhood obesity in Spain; National Health Survey 2006-2007. Nutr Hosp 2012;27(1):154-60. doi: 10.1590/S021216112012000100018

43. Sjöberg A, Moraeus L, Yngve A, Poortvliet E, Al-Ansari U, Lissner L. Overweight and obesity in a representative sample of schoolchildren - exploring the urban-rural gradient in Sweden. Obes Rev 2011;12(5):305-14. doi: 10.1111/j.1467789X.2010.00838.x

44. Thorisdóttir IE, Kristjansson AL, Sigfusdottir ID, Allegrante JP. The Landscape of Overweight and obesity in Icelandic Adolescents: Geographic Variation in Body-Mass Index Between 2000 and 2009. J Community Health 2012;37(1):234-41. doi: 10.1007/s10900-011-9441-z

Como citar: PASA, Luisa et al. Geolocalização de casos de sobrepeso/obesidade e pressão arterial alterada em escolares. Cinergis, Santa Cruz do Sul, v. 18, n. 3, maio 2017. ISSN 2177-4005. Disponível em: < https://online.unisc.br/seer/index.php/cinergis/article/view/9670>. Acesso em: 23 jun. 2017. doi:http://dx.doi.org/10.17058/cinergis.v18i3.9670. 